\title{
Diseño e implementación de una PTAR por lodos activos a escala de laboratorio, Facultad de Ciencias Espoch
}

\section{Design and implementation of a ptar for active sludge at laboratory scale, Science Faculty Espoch}

Ana María Castillo Reinoso. ${ }^{1}$, Erika Carmen Criollo Quizphi. ${ }^{2} \&$ Monica Paola Oñate Oñate. ${ }^{3}$

\section{Recibido: 10-11-2019 / Revisado: 05-12-209 /Aceptado: 26-12-2019/ Publicado: 04-01-2020}

\begin{abstract}
.
DOI: https://doi.org/10.33262/cienciadigital.v4i1.1119

Research - experimental method was applied and it consisted in checking of several studies carried out in similar conditions, sampling in the cheese Factory Llin Llin, it is located at Pucará -Colta canton using point sampling, microbiological, physical, chemical and wastewater, development of previous calculations, construction, treatment and actual calculations. The building material for the laboratory scale plant was polycarbonate, the dimensions are taken into account the $12 \%$ of those obtained in previous calculations, tha plant har three tanks: reservoir, aeration and sedimentation with the following dimensions $50 \mathrm{~cm} \times 50 \mathrm{~cm} \times 75 \mathrm{~cm}$ and two tanks: treated water storage and sludge with dimensions $50 \mathrm{~cm} \times 50 \mathrm{~cm} \times 50 \mathrm{~cm}$. The settling tank has a $20 \%$ slope. The treatment started using different hydraulic retention times (72-60-4836-24-12-8-4 hours) for each simple and 11 days of cell retention. As a result of the treatment it was accomplished when removing BOD with an initial value of $28300 \mathrm{mg} / \mathrm{L}$ to $350 \mathrm{mg} / \mathrm{L}$ of a hydraulic retention time of 4 hours. It is concluded that the biological treatment performed in the laboratory scale plant has presented satisfactory efficiency of $98 \%$ for sewage of cheese factories. It is recommended to develop a strict control on the $\mathrm{pH}$, temperature, dissolved oxygen, settleable solids, suspended and volatile to ensure proper treatment and reduce biochemical oxygen demanded.
\end{abstract}

Keywords: Laboratory, Treatment, samples, biological, organic

\footnotetext{
${ }^{1}$ Escuela Superior Politécnica del Chimborazo, Facultad de Ciencias; Riobamba Ecuador ana.castillo@espoch.edu.ec

2 Ingeniera en Biotecnología Ambiental, Riobamba Ecuador, eryswt@gmail.com

${ }^{3}$ Universidad Politécnica Salesiana, Ing. Ambiental, monate@ est.ups.edu.ec
} 


\section{Resumen.}

Se utilizó el método investigativo-experimental que consistió en: investigación de varios trabajos realizados en circunstancias similar, de la quesera Llin Llin Pucará Cantón Colta manejando muestreo puntual, precisión física, química y microbiológica del agua residual, preparación de cálculos previos, construcción, procedimiento y cálculos reales. La construcción elaborada para la planta a graduación laboratorio fue policarbonato, las extensiones han sido tomadas con fundamento el $12 \%$ de las obtenidas en cómputos previos, la planta cuenta con tres tanques: reservorio, aireación y sedimentación de dimensiones $50 \mathrm{~cm}$ x $50 \mathrm{~cm}$ x $75 \mathrm{~cm}$ y dos depósitos: provisión de agua tratada y lodos con dimensiones $50 \mathrm{~cm} \times 50 \mathrm{~cm}$ x $50 \mathrm{~cm}$. El tanque sedimentador enseña una inclinación del 20\%. El método inició usando otros tiempos de suspensión hidráulica (72-60-48-36-24-12-8-4 horas) para cada muestra y 11 días de retención celular. resultado del procedimiento se logró la separación de demanda bioquímica de oxígeno (DBO) con un valor inicial de $28300 \mathrm{mg} / \mathrm{L}$ a $350 \mathrm{mg} / \mathrm{L}$ en un tiempo de retención hidráulica de 4 horas. Se concluye que el método biológico realizado en la planta a escala laboratorio es satisfactorio mostrando una eficiencia del $98 \%$ para aguas residuales de quesera. Se confía realizar un exacto control en cuanto al $\mathrm{pH}$, temperatura, oxígeno disuelto, sólidos sedimentables, suspendidos y volátiles para certificar un adecuado sistema y reducir la demando bioquímica de oxígeno formidablemente.

Palabras claves. Laboratorio, Tratamiento, muestras, biológico, orgánicos.

\section{Introducción.}

En la actualidad una de las mayores preocupaciones a nivel mundial es la conservación del ambiente, y uno de los problemas que más preocupa a la humanidad es la gran cantidad de aguas residuales que sin un tratamiento previo son vertidas a los cuerpos de agua, de modo que en los últimos se ha empezado a desarrollar distintos tipos de tratamiento para el agua residual. El tratamiento biológico ha probado ser uno de los más eficientes, el cual se basa en la capacidad que tienen los microorganismos para metabolizar y convertir la materia orgánica en suspensión y disuelta, en tejido celular nuevo y diferentes gases.

Dentro de este tipo de tratamiento el oxígeno desempeña un papel primordial debido a que su ausencia o presencia condiciona el tipo de microorganismos que se encargaran de degradar y eliminar la materia orgánica que se presente en el agua residual.

El tratamiento de aguas residuales mediante lodos activados se considera como un método de tratamiento biológico aerobio en suspensión, su nombre proviene de la producción de una masa activada de microorganismos contenidos en un reactor capaces de metabolizar y consumir la materia orgánica presente en el agua residual en un medio aerobio.

Al poseer las aguas residuales tanto domésticas como industriales una gran variedad de microorganismos capaces de remover materia orgánica, patógenos y nutrientes (Nitrógeno y Fósforo), el empleo del tratamiento por lodos activados ofrece una buena alternativa para su depuración. (Castillo, 2014) 
Una planta de lodos activados es un sistema de mezcla completa, el ambiente aerobio en el reactor se consigue mediante el uso de aireadores mecánicos que pueden ser colocados ya sea en el lecho o superficie del mismo. Al transcurrir un periodo determinado de tiempo la mezcla líquida, aguas residuales con floc biológico en suspensión, es separada en un sedimentador y parte de las células sedimentadas se recirculan con el fin de mantener en el reactor la concentración de células deseadas, mientras que la otra parte se purga del sistema y se descarga el efluente clarificado.

Por todos los beneficios que este tipo de tratamiento presenta, en la actualidad se ha optado por el desarrollo de modelos de plantas de tratamiento de aguas residuales a escala piloto o de laboratorio con la finalidad de obtener parámetros tanto para el diseño como para la operación de los sistemas de tratamiento, ya que generalmente las plantas a escala mayor presentan problemas de operación y funcionamiento como consecuencia de diseños inapropiados basados en parámetros que no corresponden a las características físico-químicas del efluente, así como a las condiciones ambientales del lugar en donde se genera el mismo. (Castillo, 2014)

\section{Planteamiento del Problema}

El agua excedente fruto de las actividades de los seres humanos al no recibir el manejo anterior moderadas derechamente en organismos $\mathrm{u}$ orígenes de aguas nativos se bautizan en una amenaza difícil tanto para el ambiente como para la salud humana, ya que estas aguas muestran admisiones concentraciones de material orgánica, sólidos suspendidos, nutrientes y patógenos.

\section{Justificación de la Investigación}

Al ser el agua uno de los recursos empleado por todos quienes habitamos el planeta, a diario se generan millones de litros de agua residual en el mundo lo cual es inevitable ya que son producto de todas las actividades de los seres humanos tanto domésticas como industriales, estas aguas al no recibir un tratamiento previo y ser descargadas directamente en cuerpos o fuentes de aguas naturales se convierten en una amenaza grave tanto para el ambiente como para la salud humana, ya que estas aguas presentan altas concentraciones de materia orgánica, sólidos suspendidos, nutrientes y patógenos.

Por lo tanto, con el fin de preservar el medio ambiente, cuidar el recurso hídrico y proteger así la salud pública el desarrollo e implementación de procesos de tratamiento de aguas residuales se ha vuelto indispensable. Siendo actualmente los procesos biológicos los más utilizados dentro de los cuales tenemos el procedimiento por lodos activos.

Siendo el objetivo principal el diseñar, implementar y poner en funcionamiento una planta de depuración de aguas residuales por lodos activos a escala de laboratorio en la Facultad de Ciencias de la ESPOCH con la finalidad de que esta sea empleada para obtener parámetros de diseño y operación que definan las dimensiones y comportamiento de este tipo de tratamiento, además obtener información de que tan beneficioso es su aplicación para determinados tipos de aguas residual, de modo que pueda ser llevado a escala mayor contribuyendo así a la disminución de la carga contaminante de las aguas residuales generadas a diario logrando mantenerlas dentro de los límites establecidos por la norma (TULAS). (Castillo, 2014) 


\section{Marco Teórico}

\section{Agua Residual}

Se denomina aguas residuales a todas aquellas que han sido utilizadas para diversos fines, cuyo destino son las cloacas y son transportadas mediante un sistema de alcantarillado (Romero, 2014)

Aguas residuales domésticas se considera a todos los líquidos que provienen diversos lugares como: viviendas, edificios comerciales e institucionales. Aguas residuales municipales se conoce a los residuos líquidos que son transportados por el sistema alcantarillado y tratados en una planta de tratamiento municipal. Mientras que a las aguas que se generan de las descargas de industrias se conoce como aguas residuales industriales (Romero, 2014)

\section{Características Físicas}

\section{Olores}

Los olores se producen debido a la descomposición de la materia orgánica y a los gases que producto de este proceso son liberados. El agua residual que ha sido descargada recientemente tiene un olor propio, a pesar de ser desagradable, resulta más tolerable que el color del agua residual séptica. En el agua residual séptica, la acción de microorganismos anaerobios, los cuales producen sulfuro de hidrógeno da el olor propio a la misma, esto ocurre al reducirse los sulfatos a sulfitos.

Al momento de realizar instalaciones de plantas tratamiento de aguas residuales, se presenta un gran inconveniente ya que genera el rechazo por parte de poblaciones cercanas debido a los olores que se generan. (EDDY, T CALF Y, 2012)

Cuando los olores generados por las plantas de tratamiento son fuertes, varios son los problemas sobre los humanos como: menor consumo de agua, pérdida del apetito, dificultades respiratorias, náuseas, vómito, perturbaciones mentales, deterioro de las relaciones humanas, pérdida del potencial de desarrollo. (Romero, 2014, pág. 23)

\section{Temperatura}

Por lo general el agua residual suele presentar una temperatura más elevada que el agua de suministro, debido a la incorporación de aguas de diversas fuentes o de diferentes usos las cuales son más calientes. Las temperaturas que se registran durante la mayor parte del año en las aguas residuales son más altas que le agua normal, y solo son menores cuando se presentan climas calurosos, esto se debe a que el calor específico del agua es mucho mayor que la del aire. (EDDY, T CALF Y, 2012)

\section{Microorganismos}

El predominio de protozoos, rotíferos y gusanos como los nematodos, está relacionada con la calidad del lodo activado, con los parámetros operativos y la edad del lodo, así como también es importante saber que los protozoos y los rotíferos son organismos depuradores del agua residual en donde los primeros consumen bacterias dispersas no loculadas y los segundos, partículas de floc biológico suspendidas. (Viracucha, 2012) 


\section{Contaminantes de importancia en el tratamiento del agua residual}

La mayoría de normas establecidas nacional e internacionalmente exigen la eliminación de nutrientes y de los contaminantes prioritarios. Si se desea utilizar nuevamente el agua residual, las normativas son más exigentes, se debe eliminar por completo compuestos orgánicos refractarios, metales pesados y, en algunos casos, sólidos inorgánicos disueltos (usal, 2013)

\section{Características Químicas}

\section{Materia Orgánica}

Son sólidos que provienen del reino animal y vegetal, así como de actividades humanas relacionadas con la síntesis de compuestos orgánicos.

Como principales sustancias orgánicas que se pueden encontrar en el agua residual están: las proteínas (40-60\%), los hidratos de carbono (25-50\%), y las grasas y aceites (10\%). La urea es otro compuesto orgánico con importante presencia en el agua residual. Sin embargo, la velocidad del proceso de descomposición de la urea es relativamente lenta por lo que es poco común que esté presente en las aguas residuales que no sean muy recientes. (EDDY, T CALF $\mathrm{Y}, 2012)$

\section{Características Biológicas}

En las aguas residuales se hacen presentes grandes cantidades de microorganismos (algunos de ellos son patógenos). Entre los principales se debe mencionar al virus de la Hepatitis, coliformes, entre otros. (BORJA, 2011)

La mayoría de los desórdenes intestinales están relacionados a las enfermedades provocadas por bacterias patógenas transportadas por el agua. (Valdez, 2003)

\section{Detergentes}

Cuando los detergentes entran en contacto con el agua perturban su tensión superficial y provocan la formación de burbujas, esto se ocasiona gracias a su contenido de agentes superficiales activos o surfactantes las cuales son sustancias que se combinan en una sola molécula un grupo hidrofóbico con un hidrofílico, (gobierno, 2014)

\section{Metodología de Análisis}

\section{Parte Experimental, lugar de investigación}

La ejecución del presente proyecto de investigación tuvo lugar en el laboratorio de Análisis Instrumental de la Facultad de Ciencias, en la Escuela Superior Politécnica de Chimborazo. Mientras que la caracterización de las muestras de agua residual durante el proceso de tratamiento se lo realizó en el laboratorio de Análisis Técnicos de la Facultad de Ciencias.

\section{Lugar de muestreo}

El lugar seleccionado para la toma de las muestras de agua residual con las cuales se llevó a cabo los diferentes ensayos requeridos en este proyecto fue la "Quesera Llin Llin Pucará", localizada en el cantón Colta, parroquia de Columbe en la Comunidad de Llin Llin Pucará, la cual viene funcionando desde el año 2010 con el financiamiento de la Cruz Roja Española y la Fundación La Caixa, su producción es de aproximadamente 200 quesos diarios en presentaciones de 500 y $900 \mathrm{~g}$. 


\section{Caracterización del agua residual previo al diseño de la planta.}

El agua residual proveniente de la Quesera "Llin Llin Pucara" fue caracterizada física, química y microbiológicamente a fin de conocer la concentración de contaminantes que esta posee, para lo cual se procedió de la siguiente manera:

\section{Obtención de la muestra de agua residual para los análisis físicos, químicos y microbiológicos}

Para la recolección de la muestra de agua residual se empleó el método de muestreo puntual, ya que se procedió a tomar del punto de descarga de la quesera 2 litros del agua residual en un recipiente previamente purgado, esta muestra fue empleada para los respectivos análisis físicos y químicos. Mientras que para el análisis microbiológico la muestra fue recogida en un frasco estéril, evitando así alguna alteración en los resultados.

Dichas muestras fueron llevadas inmediatamente al laboratorio de Análisis instrumental de la ESPOCH, en donde se llevó a cabo cada uno de los análisis.

\section{Diseño de la planta a escala de laboratorio}

El boceto de la planta de método de agua residual por lodos activos a escala de laboratorio se basó en la caracterización previa del agua, a partir de lo cual empleando las ecuaciones y valores típicos de diseño citados en el punto 1.1.5.7 del marco teórico se realizaron los cálculos proporcionados a DBO del efluente, biomasa en el reactor, volumen del reactor, tiempo medio de retención hidráulica, tiempo medio de retención celular, producción de lodo, producción de sólidos totales de desecho, caudal de lodos de desecho, caudal de recirculación, relación de recirculación, carga orgánica volumétrica, relación alimento microorganismo, demanda de oxígeno, caudal de aire en condiciones normales, caudal de aire real, volumen de aire requerido por unidad de DBO, volumen de aire requerido por unidad de DBO removida, eficiencia en remoción de DBO total, eficiencia en remoción de DBO soluble, tasa específica de utilización de sustrato, y dimensiones del sedimentador secundario; todo esto con el fin de determinar los diversos parámetros de diseño con los cuales será construida la planta. Además, para el diseño se consideró un caudal de $200 \mathrm{~L} / \mathrm{h}$.

\section{Construcción de la planta a escala de laboratorio.}

A partir de los valores obtenidos en el diseño se decidió considerar el 12\% de las dimensiones totales calculadas para la construcción del equipo; dicha decisión se la tomó por fines didácticos, económicos y de espacio. Antes de la construcción se elaboró un plano en el cual constan los tanques con los que contará la planta con sus respectivas dimensiones, como se muestra a continuación:

\section{Pruebas de control del funcionamiento de la planta}

Antes de iniciar con el tratamiento se realizaron pruebas con agua potable con el fin de verificar el correcto funcionamiento de cada uno de los equipos instalados, es así que al colocar el agua dentro de los tanques se pudo observar una expansión de los mismos y seguido a esto una filtración del agua producto de la presión ejercida por esta, de modo que se tomaron las medidas respectivas para solucionar dichos inconvenientes. 
Tabla 1: Parámetros caracterizados en los lodos

\begin{tabular}{ccc}
\hline Parámetro & Unidades & Método \\
\hline Sólidos totales & $\mathrm{mg} / \mathrm{L}$ & Gravimétrico \\
$\mathrm{N}$ & $\%$ & Olsen modificado \\
$\mathrm{P}$ & $\%$ & Olsen modificado \\
$\mathrm{K}$ & $\%$ & Olsen modificado \\
\hline
\end{tabular}

Fuente: Métodos RELASE Ecuador

Muestreo para el control de variables durante el tratamiento

EL propósito de conservar un control del desarrollo del procedimiento del agua residual se procedió a tomar muestras del tanque de aireación, así como del efluente, dichas muestras fueron colectadas en recipientes previamente purgados y en recipientes estériles, las mismas que fueron empleadas para efectuar el análisis mencionado

\section{Determinación de los coeficientes cinéticos en reactores de mezcla completa}

El medio protegido por Metcalf y Eddy involucra la operación de la planta piloto en diferentes tiempos de retención, por lo menos para cinco valores diferentes, dentro de un intervalo de uno a diez días (1).

Los valores a registrar serán los siguientes:

\section{Cálculos de diseño}

\section{Cálculos preliminares para el diseño de la planta de tratamiento}

Para el diseño de la planta de tratamiento por lodos activos a escala de laboratorio se emplearon los siguientes datos:

Tabla 2. Datos para determinación de parámetros de diseño

\begin{tabular}{ccc}
\hline PARÁMETRO & VALOR & UNIDADES \\
\hline DBO afluente & 28300 & $\mathrm{mg} / \mathrm{L}$ \\
SSV entrada & 13360 & $\mathrm{mg} / \mathrm{L}$ \\
Sólidos totales entrada & 20300 & $\mathrm{mg} / \mathrm{L}$ \\
SST entrada & 18558 & $\mathrm{mg} / \mathrm{L}$ \\
\hline
\end{tabular}

Fuente: Grupo de Investigación.

Tabla 3. Datos para determinación de parámetros de diseño

\begin{tabular}{ccc}
\hline PARÁMETRO & VALOR & UNIDADES \\
\hline DBO efluente & 7075 & $\mathrm{mg} / \mathrm{L}$ \\
SST salida & 4639.5 & $\mathrm{mg} / \mathrm{L}$ \\
\hline
\end{tabular}

Fuente: Grupo de Investigación.

Para los valores presentados en la tabla $\mathrm{X}$ se consideró un $75 \%$ de la eficiencia de la planta. 
Tabla 4.- Datos para determinación de parámetros de diseño

\begin{tabular}{ccc}
\hline PARÁMETRO & VALOR & UNIDADES \\
\hline \multirow{3}{*}{ Caudal afluente } & 200 & $\mathrm{~L} / \mathrm{h}$ \\
& 4800 & $\mathrm{~L} / \mathrm{d}$ \\
& 3.34 & $\mathrm{~L} / \mathrm{min}$ \\
& 4.8 & $\mathrm{~m}^{3} / \mathrm{d}$ \\
\hline
\end{tabular}

Fuente: Grupo de Investigación.

El caudal considerado corresponde al 50\% del caudal teórico de las cabezas de poder empleadas

Tabla 5. Datos para determinación de parámetros de diseño

\begin{tabular}{ccc}
\hline PARÁMETRO & VALOR & UNIDADES \\
\hline SSVLM & 4000 & $\mathrm{mg} / \mathrm{L}$ \\
@c & 11 & $\mathrm{D}$ \\
Concentración de sólidos & 15000 & $\mathrm{mg} / \mathrm{L}$ \\
totales en el lodo sedimentado & & \\
Y (20 $\left.{ }^{\circ} \mathbf{C}\right)$ & $\mathbf{0 . 4 - 0 . 8}$ & $\mathbf{m g ~ V S S} / \mathbf{m g ~ D B O 5}$ \\
$\mathbf{K d ~}\left(\mathbf{2 0}^{\circ} \mathbf{C}\right)$ & $\mathbf{0 . 0 2 5 - 0 . 0 7 5}$ & $\mathbf{d}^{-\mathbf{1}}$ \\
\hline
\end{tabular}

Fuente: Metcalf y Eddy

\section{DBO del efluente}

Para la determinación de la DBO del efluente se empleará la ecuación 2.

$\mathrm{S}_{\mathrm{e}}=\mathrm{DBO}_{\mathrm{e}}-(0.63) \mathrm{S} \mathrm{S} \mathrm{S}_{\mathrm{e}}=7075 \frac{\mathrm{mg}}{\mathrm{L}}-(0.63)\left(4639.5 \frac{\mathrm{mg}}{\mathrm{L}}\right) \quad \mathrm{S}_{\mathrm{e}}=4152.115 \mathrm{mg} / \mathrm{L}$

\section{Biomasa en el reactor}

En base a la ecuación 3 tenemos

$\mathrm{XV}=\frac{\Theta_{\mathrm{C}} \mathrm{Y} \mathrm{Q}\left(\mathrm{So}-\mathrm{S}_{\mathrm{e}}\right)}{1+\mathrm{k}_{\mathrm{d}} \theta_{\mathrm{C}}} \quad \mathrm{XV}=\frac{(11 \mathrm{~d})(0,6 \mathrm{mg} \mathrm{SSV} / \mathrm{mg} \mathrm{DBO})\left(4800 \frac{\mathrm{L}}{\mathrm{d}}\right)\left(28300 \frac{\mathrm{mg}}{\mathrm{L}}-4152.115 \frac{\mathrm{mg}}{\mathrm{L}}\right)}{1+\left(0.06 \mathrm{~d}^{-1)}(11 \mathrm{~d})\right.}$

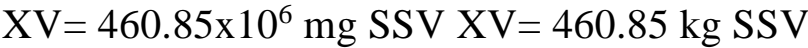

\section{Volumen del reactor}

Para conocer el volumen del reactor se empleó la ecuación 4.

$\mathrm{V}=\frac{\mathrm{XV}}{\mathrm{X}}$

$\mathrm{V}=\frac{460.85 \times 106 \mathrm{mg} \mathrm{SSV}}{4000 \mathrm{mg} / \mathrm{L}}$ 
$\mathrm{V}=115212.5 \mathrm{~L}$

$\mathrm{V}=115.21 \mathrm{~m}^{3}$

Con lo cual se determina que el tanque de aireación tendrá las siguientes medidas: $4.25 \mathrm{~m}$ de largo, $4.25 \mathrm{~m}$ de ancho y $6.5 \mathrm{~m}$ de profundidad.

\section{Tiempo medio de retención hidráulica}

Para la determinación del tiempo que el agua residual permanecerá en el tanque de aireación nos basamos en la ecuación 5 .

$\tau=\frac{\mathbf{v}}{\mathbf{Q}}$

$\tau=\frac{115212.5 \mathrm{~L}}{3.34 \mathrm{~L} / \mathrm{min}}$

$\tau=34494.76 \mathrm{~min}$

$\tau=23.95 \mathrm{~d}$

Caudal de los lodos de desecho

$\mathrm{Q}_{\mathrm{W}}=\frac{\text { lodos seco* }\left(10^{3}\right)}{\text { concentración de sólidos totales en el lodo sedimentado }} \quad \mathrm{Q}_{\mathrm{W}}=\frac{52.36 \mathrm{kgSSV} / \mathrm{d} *\left(10^{3}\right)}{15000 \mathrm{mg} / \mathrm{L}}$

$\mathrm{Q}_{\mathrm{w}}=3.49 \mathrm{~m}^{3} / \mathrm{d} \quad \mathrm{Q}_{\mathrm{w}}=3490 \mathrm{~L} / \mathrm{d} \quad \mathrm{Q}_{\mathrm{w}}=2.42 \mathrm{~L} / \mathrm{min}$

\section{Caudal de recirculación}

Se empleará la ecuación 10.

$\mathrm{Q}_{\mathrm{R}}=\frac{\mathrm{QX}}{\mathrm{X}_{\mathrm{R}}-\mathrm{X}}$

Se considerará que el $80 \%$ de los sólidos son volátiles dentro del lodo sedimentado.

$\mathrm{Q}_{\mathrm{R}}=\frac{3.34 \frac{\mathrm{L}}{\mathrm{min}}\left(4000 \frac{\mathrm{mg}}{\mathrm{L}}\right)}{\left(0,8 * 15000 \frac{\mathrm{mg}}{\mathrm{L}}\right)-4000 \mathrm{mg} / \mathrm{L}} \quad \mathrm{Q}_{\mathrm{R}}=1.67 \mathrm{~L} / \mathrm{min} \mathrm{Q}_{\mathrm{R}}=2404.8 \mathrm{~L} / \mathrm{d} \quad \mathrm{Q}_{\mathrm{R}}=1.67 \times 10^{-3} \mathrm{~m}^{3} / \mathrm{min}$

\section{Relación de recirculación}

Se empleará la ecuación 11.

$\mathrm{R} \%=\frac{\mathrm{Q}_{\mathrm{R}}}{\mathrm{Q}} \quad \mathrm{R} \%=\frac{1.67 \mathrm{~L} / \mathrm{min}}{3.34 \mathrm{~L} / \mathrm{min}} \quad \mathrm{R}=0.5=50 \% \quad \mathrm{~g} / \mathrm{min}^{3}$

Relación alimento microorganismo. Esta relación se la determina en base a la ecuación 14.

$\frac{\mathrm{A}}{\mathrm{M}}=\frac{\mathrm{Q} * \mathrm{So}}{\mathrm{VX}} \quad \frac{\mathrm{A}}{\mathrm{M}}=\frac{\left(4.8 \frac{\mathrm{m}^{3}}{\mathrm{~d}}\right)\left(28300 \frac{\mathrm{mg}}{\mathrm{L}}\right)}{\left(115.21 \mathrm{~m}^{3}\right)\left(4000 \frac{\mathrm{mg}}{\mathrm{L}}\right)} \quad \frac{\mathbf{A}}{\mathbf{M}}=0.29 \mathrm{~d}^{-1}$ 
Demanda de oxígeno

Para este parámetro se determina en base a la ecuación 15.

$$
\begin{aligned}
& \mathrm{DO}=1.5 \mathrm{Q}\left(\mathrm{S}_{\mathrm{o}}-\mathrm{S}_{\mathrm{e}}\right)-1.42 \mathrm{X}_{\mathrm{r}} \mathrm{Q}_{\mathrm{w}} \\
& \mathrm{DO}=1.5(3.34 \mathrm{~L} / \mathrm{min})\left(28300 \frac{\mathrm{mg}}{\mathrm{L}}-\frac{41152.115 \mathrm{mg}}{\mathrm{L}}\right)-1.42(0.8 * 15000)\left(2.42 \frac{\mathrm{L}}{\mathrm{min}}\right) \\
& \mathrm{DO}=79744.10 \mathrm{mg} / \mathrm{min} \quad \mathrm{DO}=114.83 \mathrm{~kg} / \mathrm{d}
\end{aligned}
$$

Caudal de aire en condiciones normales

$\mathrm{Q}_{\text {aire }}=\frac{\text { Do }}{(0.232)(1.20)} Q_{\text {aire }}=\frac{114.83 \mathrm{~kg} / \mathrm{d}}{(0.232)(1.20)} Q_{\text {aire }}=412.46 \mathrm{~m}^{3} / \mathrm{d} \quad Q_{\text {aire }}=412.46 \times 10^{3} \mathrm{~L} / \mathrm{d}$

\section{Caudal de aire real}

Se utilizará la ecuación 17.

$Q_{\text {aire real }}=\frac{Q_{\text {aire }}}{0.08} \quad Q_{\text {aire real }}=\frac{412.46 \mathrm{~m}^{3} / \mathrm{d}}{0.08}$

Qaire real $=5155.75 \mathrm{~m}^{3} / \mathrm{d}$ Qaire real $=5155801.006 \mathrm{~L} / \mathrm{d}$

Volumen de aire requerido por unidad de DBO aplicada al tanque de aireación

Se empleará la ecuación 18.

$\frac{Q_{\text {aire }}}{\text { DBO }}=\frac{Q_{\text {aire real }(1000)}}{S_{o} Q} \frac{Q_{\text {aire }}}{\text { DBO }}=\frac{5155801.006 \frac{\mathrm{L}}{\mathrm{d}}(1000)}{(28300) \mathrm{mg} / \mathrm{L}\left(4800 \frac{\mathrm{L}}{\mathrm{d}}\right)} \frac{\mathrm{Q}_{\text {aire }}}{D B O}=37.95 \mathrm{~m}^{3}$ aire $/ \mathrm{kg}$ DBO

\section{Volumen de aire requerido por unidad de DBO removida}

Para el cálculo se empleará la ecuación 19.

$\frac{Q_{\text {aire }}}{\text { DBO }}=\frac{Q_{\text {aire real }}(1000)}{\left(S_{0}-S_{e}\right) Q} \frac{Q_{\text {aire }}}{\text { DBO }}=\frac{5155801.006 \frac{\mathrm{L}}{\mathrm{d}}(1000)}{(28300-4152.115) \frac{\mathrm{mg}}{\mathrm{L}}\left(4800 \frac{\mathrm{L}}{\mathrm{d}}\right)} \frac{\mathrm{Q}_{\text {aire }}}{\text { DBO }}=44.48 \mathrm{~m}_{\text {aire }}^{3} / \mathrm{kg}$ DBO

\section{Eficiencia en remoción de DBO total}

La eficiencia se determinó en base a la ecuación 20 .

$\mathrm{E}=\frac{\left(\mathrm{S}_{\mathrm{o}}-\mathrm{S}_{\mathrm{e}}\right)}{\mathrm{S}_{\mathrm{o}}} \quad \mathrm{E}=\frac{(28300-7075) \mathrm{mg} / \mathrm{L}}{28300 \mathrm{mg} / \mathrm{L}} \quad=75 \%$

\section{Eficiencia en remoción de DBO soluble}

Dicha eficiencia se determinó en base a la ecuación 21 .

$\mathrm{E}=\frac{\left(\mathrm{S}_{\mathrm{o}}-\mathrm{S}_{\mathrm{e}}\right)}{\mathrm{S}_{\mathrm{o}}} \quad \mathrm{E}=\frac{(28300-4152.115) \mathrm{mg} / \mathrm{L}}{28300 \mathrm{mg} / \mathrm{L}} \quad \mathrm{E}=85 \%$

\section{Tasa específica de utilización del sustrato}

Este cálculo se basa en la ecuación 22.

$\mathrm{U}=\frac{(\mathrm{So}-\mathrm{S})}{\tau \mathrm{X}}=\frac{\mathrm{Q}(\mathrm{So}-\mathrm{S})}{\mathrm{VX}} \mathrm{U}=\frac{3.34 \mathrm{~L} / \mathrm{min} *(28300-7075) \mathrm{mg} / \mathrm{L}}{115212.5 \mathrm{~L}\left(\frac{4000 \mathrm{mg}}{\mathrm{L}}\right)} \mathrm{U}=1.54 \times 10^{-4} \mathrm{~min}^{-1} \mathrm{U}=0.22 \mathrm{~d}^{-1}$

Diseño del sedimentador 
Para el diseño del sedimentador se consideró el mismo volumen y área del tanque de aireación.

\section{Tasa superficial}

La tasa superficial se determinó según la ecuación 23.

$\mathrm{TS}=\frac{\mathrm{Q}}{\mathrm{A}} \quad \mathrm{TS}=\frac{4.8 \mathrm{~m}^{3} / \mathrm{d}}{18 \mathrm{~m}^{2}} \quad \mathrm{TS}=0.27 \mathrm{~m}^{2} / \mathrm{m}^{3} \mathrm{~d}$

\section{Carga de sólidos}

Según la ecuación 24 tenemos

$\mathrm{CS}=\frac{\left(\mathrm{Q}+\mathrm{Q}_{\mathrm{R}}\right) * \mathrm{X}_{\mathrm{T}}}{\mathrm{A} * 1000 * 24} \quad \mathrm{CS}=\frac{\left(4.8 \mathrm{~m}^{3} / \mathrm{d}^{+2.4048} \mathrm{~m}^{3} / \mathrm{d}\right) * 4000 \mathrm{mg} / \mathrm{L}}{18 \mathrm{~m}^{2} * 1000 * 24} \quad \mathrm{Cs}=0.07 \mathrm{~kg} \mathrm{SS} / \mathrm{m}^{2} \mathrm{~h}$

\section{Pendiente del tanque de sedimentación (Autores)}

$\mathrm{P}=$ Determinación de caudales

A continuación, se determinarán cada uno de los caudales necesarios dentro de la planta de tratamiento de agua residual por lodos activos a escala de laboratorio, lo cual se realizará en base a la ecuación $1 . Q=\frac{\mathrm{V}}{\mathrm{t}}$

Para lo cual se considerará el tiempo que tardo en llenarse de agua un recipiente de volumen determinado.

\section{Caudal del paso del agua tanque reservorio - tanque de aireación}

Tabla6. Datos para determinación de caudal

\begin{tabular}{ccc}
\hline Volumen $(\mathbf{L})$ & Tiempo $(\mathbf{s})$ & Caudal $(\mathbf{L} / \mathbf{s})$ \\
\hline 2 & 34,66 & 0,0577 \\
2 & 33,64 & 0,0594 \\
2 & 33,18 & 0,0603 \\
2 & 34,44 & 0,0581 \\
2 & 34,64 & 0,0577 \\
2 & 34,28 & 0,0583 \\
& & $\mathbf{0 . 0 5 8 5}$ \\
\hline
\end{tabular}

Fuente: Grupo de Investigación.

El caudal fue determinado con la llave de paso abierta al 50\% 
Caudal del paso del agua tanque de aireación - tanque de sedimentación

Tabla 7. Datos para determinación de caudal

\begin{tabular}{ccc}
\hline Volumen $(\mathbf{L})$ & Tiempo $(\mathbf{s})$ & Caudal $(\mathbf{L} / \mathbf{s})$ \\
\hline 2 & 41,56 & 0,0481 \\
2 & 39,66 & 0,0504 \\
2 & 40,02 & 0,0499 \\
2 & 41,43 & 0,0482 \\
2 & 41,22 & 0,0485 \\
2 & 41,65 & 0,0480 \\
\multicolumn{2}{c}{ Caudal Promedio $(\mathbf{L} / \mathbf{s})$} & $\mathbf{0 , 0 4 8 8 5}$ \\
\hline
\end{tabular}

Fuente: Grupo de Investigación.

El caudal fue determinado con la llave de paso abierta al 50\%

\section{Caudal de salida del agua tratada}

Tabla 8.- Datos para determinación de caudal'

\begin{tabular}{ccc}
\hline Volumen (L) & Tiempo (s) & Caudal (L/s) \\
\hline 2 & 48,96 & 0,0408 \\
2 & 46,66 & 0,0428 \\
2 & 48,82 & 0,0409 \\
2 & 47,43 & 0,0421 \\
2 & 47,89 & 0,0417 \\
2 & 47,65 & 0,0414 \\
Caudal Promedio (L/s) & $\mathbf{0 , 0 4 1 4}$ \\
\hline
\end{tabular}

Fuente: Grupo de Investigación.

El caudal fue determinado con la llave de paso abierta al 50\%

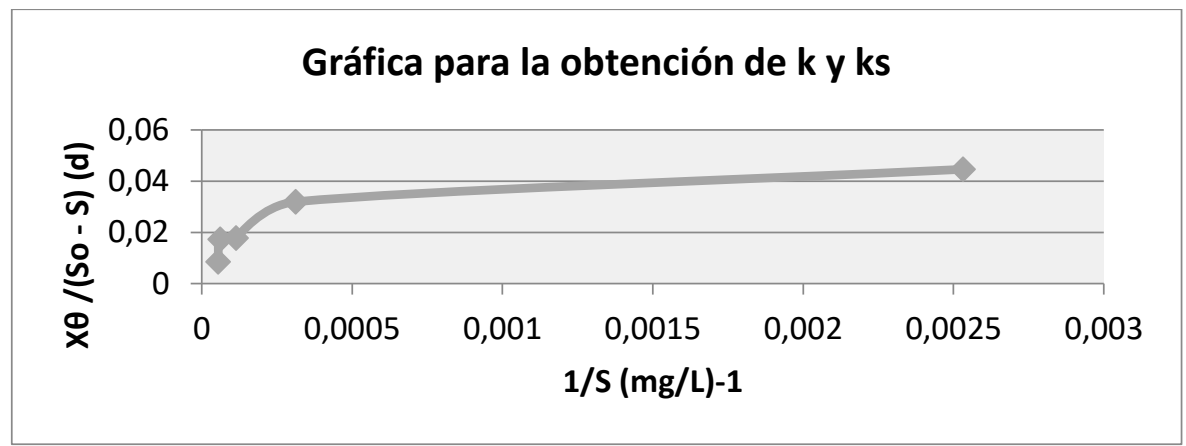

Figura 1. Obtención de ks y k (Autores)

Fuente: Grupo de Investigación.

Mediante una regresión lineal en la calculadora se tienen los siguientes valores $\frac{1}{\mathrm{k}} .=0,017 \mathrm{y} \frac{\mathrm{K}_{\mathrm{s}}}{\mathrm{k}}=11.33 \mathrm{~d} \cdot \mathrm{mg} / \mathrm{L} ;$ de donde tenemos que: 
$\mathbf{K}=\mathbf{5 8 . 8 2} \mathbf{d}^{\mathbf{1}} \quad \mathrm{Ks}=11,33 * 58,82 \quad \mathbf{K s}=\mathbf{6 6 6 , 4 3} \mathbf{~ m g} / \mathbf{L}$

A continuación, se elabora una gráfica de $\frac{1}{\theta_{c}}$ en las abscisas y $\frac{\mathrm{S}_{\mathrm{o}}-\mathrm{S}}{\mathrm{X} \theta}$ en las ordenadas, en donde la pendiente de dicha recta nos permitirá obtener el valor de $\mathrm{Y}$ y la ordenada en el origen el valor de $\mathrm{k}_{\mathrm{d}}$.



Figura 2. Obtención de kd y (Autores)

Repitiendo el procedimiento anterior mediante una regresión lineal se obtuvo los siguientes valores:

\section{$\mathrm{Kd}=\mathbf{2 , 0 4 5} \mathrm{d}^{-1} \quad \mathrm{Y}=\mathbf{0 , 1 2 1 5}(\mathrm{mg} \mathrm{SSV} / \mathrm{mg}$ DBO$)$}

Producción de sólidos totales de desecho

Para esto se empleará la ecuación 8.

Lodos seco $=\frac{\mathrm{P}_{\mathrm{x}}}{\text { porción volátil de los sólidos totales }}$ Lodos seco $=\frac{0,74 \mathrm{kgSSV} / \mathrm{d}}{0,8} \quad$ Lodos seco $=$ $0,92 \mathrm{~kg} \mathrm{SSV} / \mathrm{d}$

\section{Caudal de los Lodos de Desecho}

Nos basaremos en la ecuación 9. $\mathrm{Q}_{\mathrm{w}}=\frac{\text { lodos seco* }\left(10^{3}\right)}{\text { concentración de sólidos totales en el lodo sedimentado }}$

$\mathrm{Q}_{\mathrm{w}}=\frac{0,92 \mathrm{kgSSV} / \mathrm{d} *\left(10^{3}\right)}{47800 \mathrm{mg} / \mathrm{L}} \quad \mathrm{Q}_{\mathrm{w}}=0.02 \mathrm{~m}^{3} / \mathrm{d} \quad \mathrm{Q}_{\mathrm{w}}=19.24 \mathrm{~L} / \mathrm{d} \quad \mathrm{Q}_{\mathrm{w}}=0.01 \mathrm{~L} / \mathrm{min}$

2.1.1.1 Relación de recirculación

Se empleará la ecuación 11.

$\mathrm{R} \%=\frac{\mathrm{Q}_{\mathrm{R}}}{\mathrm{Q}} \quad \mathrm{R} \%=\frac{0,23 \mathrm{~L} / \mathrm{min}}{3.51 \mathrm{~L} / \mathrm{min}} \quad \mathrm{R} \%=6.5 \%$

\section{Índice volumétrico de lodos ivl}

Para determinar este valor usaremos la ecuación 12 .

$\mathrm{IVL}=\frac{\mathrm{V}_{30}}{\mathrm{X}_{\mathrm{T}}} * 1000 \quad \mathrm{IVL}=\frac{\left(30 \frac{\mathrm{mL}}{\mathrm{L}}\right)}{1681 \mathrm{mg} / \mathrm{L}} * 1000 \mathrm{IVL}=17.84 \mathrm{~mL} / \mathrm{g}$

\section{Carga orgánica volumétrica (cov)}

La carga orgánica volumétrica la determinaremos mediante la ecuación 13. 
$\mathrm{COV}=\frac{\mathrm{Q} \mathrm{S}_{\mathrm{O}}}{\mathrm{Vr}} \quad \mathrm{COV}=\frac{\left(3.51 \frac{\mathrm{L}}{\mathrm{min}}\right)\left(28300 \frac{\mathrm{mg}}{\mathrm{L}}\right)}{5350 \mathrm{~L}} \quad \mathrm{COV}=18.56 \mathrm{mg} / \mathrm{min} \mathrm{L}$

\section{Relación alimento microorganismo}

Esta relación se la determina en base a la ecuación 14 .

$\frac{\mathrm{A}}{\mathrm{M}}=\frac{\mathrm{Q} * \mathrm{So}}{\mathrm{VX}} \quad \frac{\mathrm{A}}{\mathrm{M}}=\frac{\left(5.0544 \frac{\mathrm{m}^{3}}{\mathrm{~d}}\right)\left(28300 \frac{\mathrm{mg}}{\mathrm{L}}\right)}{\left(5.35 \mathrm{~m}^{3}\right)\left(1500 \frac{\mathrm{mg}}{\mathrm{L}}\right)} \quad \frac{\mathrm{A}}{\mathrm{M}}=17.82 \mathrm{~d}^{-1}$

\section{Demanda de oxígeno}

Para este parámetro se determina en base a la ecuación 15.

DO $=1.5 \mathrm{Q}\left(\mathrm{S}_{\mathrm{o}}-\mathrm{S}_{\mathrm{e}}\right)-1.42 \mathrm{X}_{\mathrm{r}} \mathrm{Q}_{\mathrm{w}}$

$\mathrm{DO}=1.5(3.51 \mathrm{~L} / \mathrm{min})\left(28300 \frac{\mathrm{mg}}{\mathrm{L}}-85.4 \frac{\mathrm{mg}}{\mathrm{L}}\right)-1.42(0.8 * 47800)\left(0.01 \frac{\mathrm{L}}{\min }\right)$

$\mathrm{DO}=213.12 \mathrm{~kg} / \mathrm{d}$

Volumen de aire requerido por unidad de DBO aplicada al tanque de aireación Se empleará la ecuación 18.

$\frac{Q_{\text {aire }}}{\text { DBO }}=\frac{Q_{\text {aire real (1000) }}}{S_{\mathrm{o}} \mathrm{Q}} \quad \frac{\mathrm{Q}_{\text {aire }}}{\mathrm{DBO}}=\frac{9.5 \times 10^{6} \mathrm{~L} / \mathrm{d}(1000)}{(28300) \mathrm{mg} / \mathrm{L}\left(5054.4 \frac{\mathrm{L}}{\mathrm{d}}\right)} \frac{\mathrm{Q}_{\text {aire }}}{\mathrm{DBO}}=66.41 \mathrm{~m}^{3}$ aire $/ \mathrm{kg}$ DBO

Volumen de aire requerido por unidad de DBO removida

Para el cálculo se empleará la ecuación 19.

$\frac{Q_{\text {aire }}}{D B O}=\frac{Q_{\text {aire real }}(1000)}{\left(S_{\mathrm{o}}-S_{e}\right) Q} \quad \frac{Q_{\text {aire }}}{D B O}=\frac{9.5 \times 10^{6} \mathrm{~L} / \mathrm{d}(1000)}{(28300-85.4)\left(5054.4 \frac{\mathrm{L}}{\mathrm{d}}\right)} \quad \frac{\mathrm{Q}_{\text {aire }}}{\mathrm{DBO}}=66.62 \mathrm{~m}^{3}$ aire $/ \mathrm{kg}$ DBO

\section{Eficiencia en remoción de dbo total}

La eficiencia se determinó en base a la ecuación 20 .

$\mathrm{E}=\frac{\left(\mathrm{S}_{\mathrm{o}}-\mathrm{S}_{\mathrm{e}}\right)}{\mathrm{S}_{\mathrm{o}}} \quad \mathrm{E}=\frac{(28300-350) \mathrm{mg} / \mathrm{L}}{28300 \mathrm{mg} / \mathrm{L}} \quad \mathrm{E}=98 \%$

2.1.1.2 Eficiencia en remoción de dbo soluble

Dicha eficiencia se determinó en base a la ecuación 21 .

$\mathrm{E}=\frac{\left(\mathrm{S}_{\mathrm{o}}-\mathrm{S}_{\mathrm{e}}\right)}{\mathrm{S}_{\mathrm{o}}} \quad \mathrm{E}=\frac{(28300-85.4) \mathrm{mg} / \mathrm{L}}{28300 \mathrm{mg} / \mathrm{L}} \mathrm{E}=99 \%$

Tasa específica de utilización del sustrato

Este cálculo se basa en la ecuación 22.

$\mathrm{U}=\frac{(\text { So-S })}{\tau \mathrm{X}}=\frac{\mathrm{Q}(\text { So-S })}{\mathrm{VX}} \quad \mathrm{U}=\frac{(28300-350) \mathrm{mg} / \mathrm{L}}{1.06 \mathrm{~d}\left(\frac{1500 \mathrm{mg}}{\mathrm{L}}\right)} \mathrm{U}=17.58 \mathrm{~d}^{-1}$

\section{Tasa superficial}

La tasa superficial se determinó según la ecuación 23.

$\mathrm{TS}=\frac{\mathrm{Q}}{\mathrm{A}} \mathrm{TS}=\frac{4.22 \mathrm{~m}^{3} / \mathrm{d}}{2.25 \mathrm{~m}^{2}} \quad \mathrm{TS}=1.88 \mathrm{~m}^{2} / \mathrm{m}^{3} \mathrm{~d}$

$.048 \mathrm{~m}^{3} / \mathrm{s} \quad \mathrm{Q}=48.23 \mathrm{~L} / \mathrm{s}$ 


\section{Resultados y Discusión}

Caracterización del agua residual previo al tratamiento

Tabla11. Resultados de la caracterización antes del tratamiento

\begin{tabular}{ccc}
\hline PARÁMETRO & UNIDADES & RESULTADOS \\
\hline Ph & & 5.69 \\
Temperatura & ${ }^{\circ} \mathrm{C}$ & 25.9 \\
Oxígeno disuelto & $\mathrm{mg} / \mathrm{L}$ & 1.14 \\
Demanda química de oxígeno & $\mathrm{mg} / \mathrm{L}$ & 32000 \\
Demanda bioquímica de oxígeno & $\mathrm{mg} / \mathrm{L}$ & 28300 \\
Sólidos suspendidos & $\mathrm{mg} / \mathrm{L}$ & 18558 \\
Sólidos sedimentables & $\mathrm{ml} / \mathrm{l}$ & 3 \\
Sólidos volátiles & $\mathrm{mg} / \mathrm{L}$ & 13360 \\
Sólidos totales & $\mathrm{mg} / \mathrm{L}$ & 20300 \\
Mohos y levaduras & $\mathrm{UPC} / \mathrm{ml}$ & 5890 \\
Coliformes totales & $\mathrm{UFC} / \mathrm{ml}$ & 544000 \\
Escherichia coli. & $\mathrm{UFC} / \mathrm{ml}$ & 182000 \\
\hline
\end{tabular}

Fuente: Autores

Al caracterizar el agua residual procedente de la quesera "Llin Llin - Pucará" se puede observar que esta presenta una alta carga contaminante lo cual se debe a que el agua residual generada a lo largo del proceso es descargada directamente hacia el río más cercano sin haber recibido tratamiento previo, además gran parte de esta agua corresponde al suero de leche eliminado en la etapa de desuerado ya que solo una pequeña parte de este es comercializado para la alimentación de los animales de la zona.

\section{Diseño de la Planta}

Tabla 12.- Resultados de los cálculos de diseño

\begin{tabular}{|c|c|c|}
\hline PARÁMETRO & VALOR & UNIDADES \\
\hline \multicolumn{3}{|c|}{ CAUDAL } \\
\hline Caudal & 200 & $\mathrm{~L} / \mathrm{h}$ \\
\hline \multicolumn{3}{|c|}{ AIRE } \\
\hline Potencia del compresor & 1 & HP \\
\hline \multicolumn{3}{|c|}{$\begin{array}{l}\text { PARÁMETROS DE DISEÑO PARA EL TRATAMIENTO POR LODOS } \\
\text { ACTIVOS }\end{array}$} \\
\hline DBO del efluente & 4.152 .115 & $\mathrm{mg} / \mathrm{L}$ \\
\hline Biomasa en el reactor & 460.85 & Kg SSV \\
\hline Volumen del reactor & 115.21 & $\mathrm{~m}^{3}$ \\
\hline Ancho del reactor & 4.25 & $\mathrm{~m}$ \\
\hline Largo del reactor & 4.25 & $\mathrm{~m}$ \\
\hline Profundidad del reactor & 6.5 & $\mathrm{~m}$ \\
\hline Tiempo medio de retención hidráulica & 23.95 & $\mathrm{~h}$ \\
\hline Producción de lodo & 41.89 & $\mathrm{~kg} \mathrm{SSV} / \mathrm{d}$ \\
\hline Producción de Sólidos Totales de desecho & 52.36 & $\mathrm{Kg} \mathrm{SSV/d}$ \\
\hline Caudal de los lodos de desecho & 3490 & $\mathrm{~L} / \mathrm{d}$ \\
\hline
\end{tabular}




\begin{tabular}{lcc} 
Caudal de recirculación & 2404.8 & $\mathrm{~L} / \mathrm{d}$ \\
Relación de recirculación & 50 & $\%$ \\
Carga orgánica volumétrica & 0.82 & $\mathrm{mg} / \mathrm{min} \mathrm{L}$ \\
Relación alimento microorganismo & 0.29 & $\mathrm{~d}^{-1}$ \\
Demanda de oxígeno & 114.83 & $\mathrm{~kg} / \mathrm{d}$ \\
Caudal de aire en condiciones normales & $412.46 \times 10^{3}$ & $\mathrm{~L} / \mathrm{d}$ \\
Caudal de aire real & 5.155 .801 .006 & $\mathrm{~L} / \mathrm{d}$ \\
Volumen de aire requerido por unidad de & 37.95 & $\mathrm{~m}^{3}{ }_{\text {aire }} / \mathrm{kg} \mathrm{DBO}$ \\
DBO & & \\
Volumen de aire requerido por unidad de & 44.48 & $\mathrm{~m}^{3}{ }_{\text {aire }} / \mathrm{kg} \mathrm{DBO}$ \\
DBO removida & 75 & $\%$ \\
Eficiencia en remoción de DBO total & 85 & $\%$ \\
Eficiencia en remoción de DBO soluble & 0.22 & $\mathrm{~d}^{-1}$ \\
Tasa específica de utilización del sustrato & TANQUE SEDIMENTADOR & \\
\hline & 200 & $\mathrm{~L} / \mathrm{h}$ \\
\hline Caudal & 18 & $\mathrm{~m}^{2}$ \\
Área & 4.25 & $\mathrm{~m}$ \\
Largo & 4.25 & $\mathrm{~m}$ \\
Ancho & 6.5 & $\mathrm{~m}^{2}$ \\
Profundidad & 115 & $\mathrm{~m}^{3}$ \\
Volumen & 0.27 & $\mathrm{~m}^{2} / \mathrm{m}^{3} \mathrm{~d}$ \\
Tasa superficial & 0.07 & $\mathrm{~kg} \mathrm{SS} / \mathrm{m}^{2} \mathrm{~h}$ \\
Carga de sólidos & 20 & $\%$ \\
Pendiente & & \\
\hline
\end{tabular}

Fuente: Grupo de Investigación.

Las consecuencias de las medidas obtenidos para el boceto de la planta presentan valores altos lo cual se debe a que el caudal de $200 \mathrm{~L} / \mathrm{h}$ con el que se trabajó toca a un caudal teórico, así como también la asamblea de los contaminantes principalmente la DBO y DQO son dominantes.

\section{Desarrollo Experimental}

\section{Caudales}

Tabla 13. Resultados de los caudales

\begin{tabular}{ccc}
\hline PARÁMETRO & VALOR & UNIDADES \\
\hline CAUDALES & & \\
\hline Caudal tanque reservorio tanque de aireación & 0.0585 & $\mathrm{~L} / \mathrm{s}$ \\
Caudal tanque de aireación tanque de sedimentación & 0.04885 & $\mathrm{~L} / \mathrm{s}$ \\
Caudal de salida del agua tratada & 0.0414 & $\mathrm{~L} / \mathrm{s}$ \\
Caudal de recirculación 100\% & 0.984 & $\mathrm{~L} / \mathrm{s}$ \\
Caudal de recirculación 75\% & 0.966 & $\mathrm{~L} / \mathrm{s}$ \\
Caudal de recirculación 50\% & 0.940 & $\mathrm{~L} / \mathrm{s}$ \\
Caudal de recirculación 25\% & 0.546 & $\mathrm{~L} / \mathrm{s}$ \\
\hline
\end{tabular}

Fuente: Grupo de Investigación. 
Mientras que los caudales de recirculación se obtuvieron con la llave de paso abierta a diversos porcentajes, ya que no siempre se va a requerir un mismo caudal de recirculación y este se debe adaptar a las necesidades del proceso. Es así que para la parte experimental se empleó un caudal de $0.546 \mathrm{~L} / \mathrm{s}$ que corresponde a la llave de paso abierta al $25 \%$.

\section{Control de variables durante el tratamiento}

Tiempo de retención hidráulica 72 horas

Tabla14.- Tiempo de Retención Hidráulica de 72 horas

\begin{tabular}{ccccc}
\hline \multirow{2}{*}{$\begin{array}{c}\mathbf{N}^{\circ} \text { de } \\
\text { medicines }\end{array}$} & \multicolumn{3}{c}{ Parámetros } & \multicolumn{1}{c}{$\begin{array}{c}\text { Intervalos } \\
\text { de control }\end{array}$} \\
\cline { 2 - 4 } & $\mathbf{p H}$ & $\begin{array}{c}\text { Temperatura } \\
{ }^{\circ} \mathbf{C}\end{array}$ & OD mg/L & \\
\hline 1 & 6.12 & 16.8 & 2.4 & $\mathrm{c} / 12 \mathrm{~h}$ \\
2 & 6.08 & 17.3 & 2.1 & $\mathrm{c} / 12 \mathrm{~h}$ \\
3 & 5.13 & 17.8 & 1.9 & $\mathrm{c} / 12 \mathrm{~h}$ \\
4 & 5.18 & 17.5 & 2.5 & $\mathrm{c} / 12 \mathrm{~h}$ \\
5 & 5.48 & 17.7 & 2.6 & $\mathrm{c} / 12 \mathrm{~h}$ \\
6 & 6.20 & 17.3 & 2.1 & $\mathrm{c} / 12 \mathrm{~h}$ \\
Promedios & $\mathbf{5 . 6 9}$ & $\mathbf{1 7 . 4}$ & $\mathbf{2 . 2 7}$ & \\
\hline
\end{tabular}

Fuente: Grupo de Investigación.



Figura 4. Variación de pH en las 72 horas de TRH (Autores)

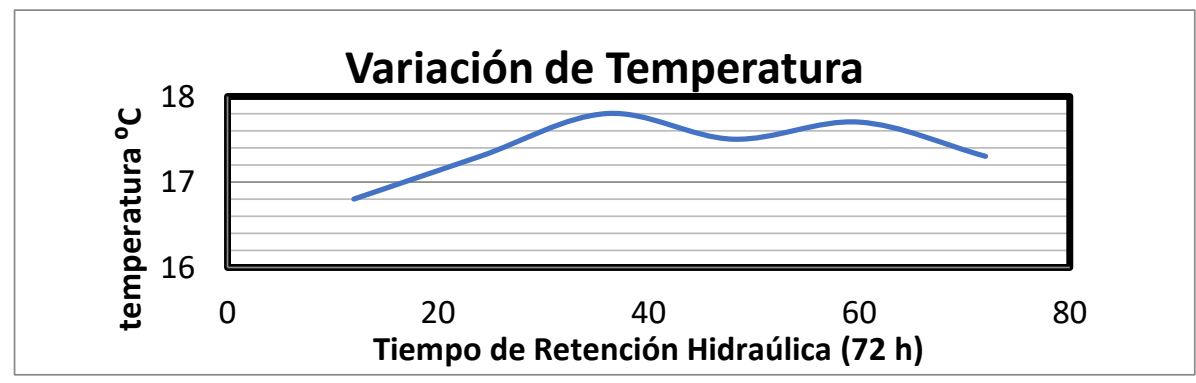

Figura 5. Variación de temperatura en las 72 horas de TRH (Autores) 




Figura 6. Variación de Oxígeno Disuelto en las 72 horas de TRH (Autores)

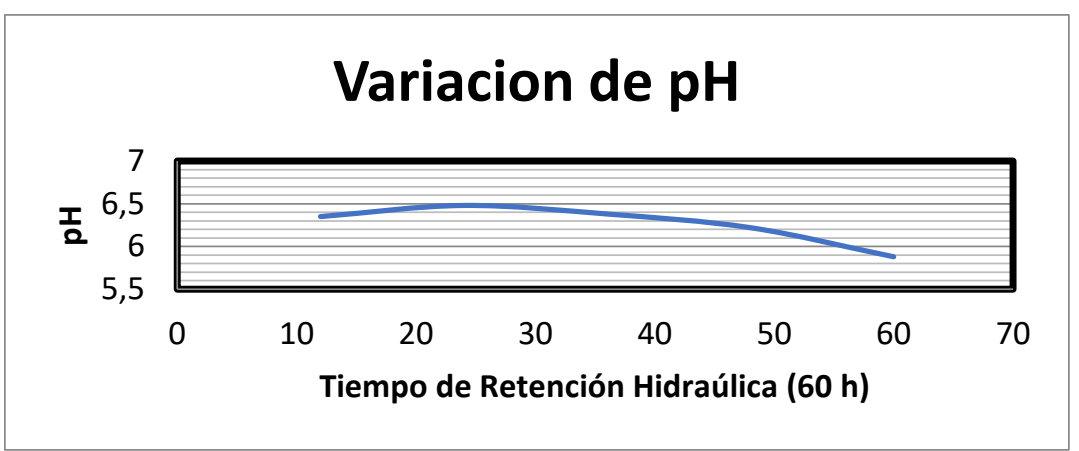

Figura7. Variación de pH en las 60 horas de TRH (Autores)

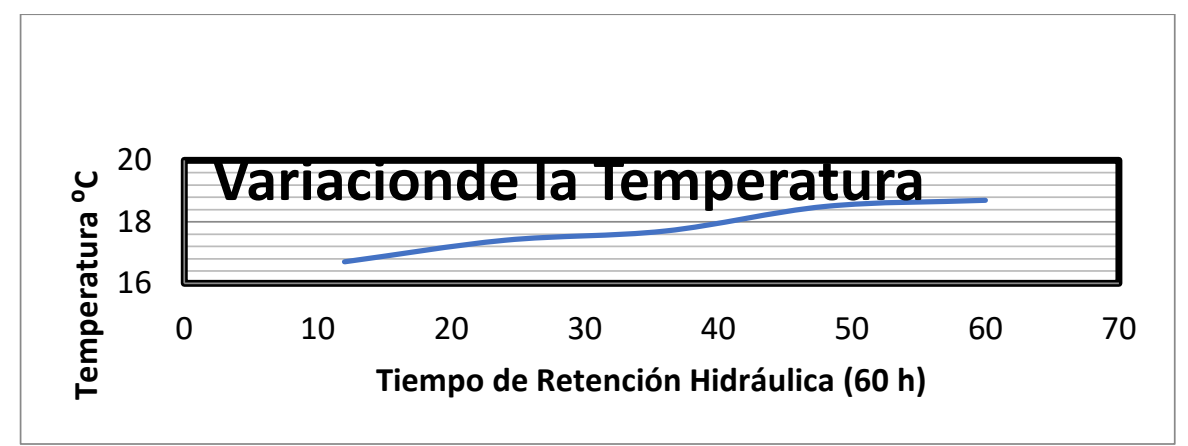

Figura8. Variación de temperatura en las 60 horas de TRH (Autores)

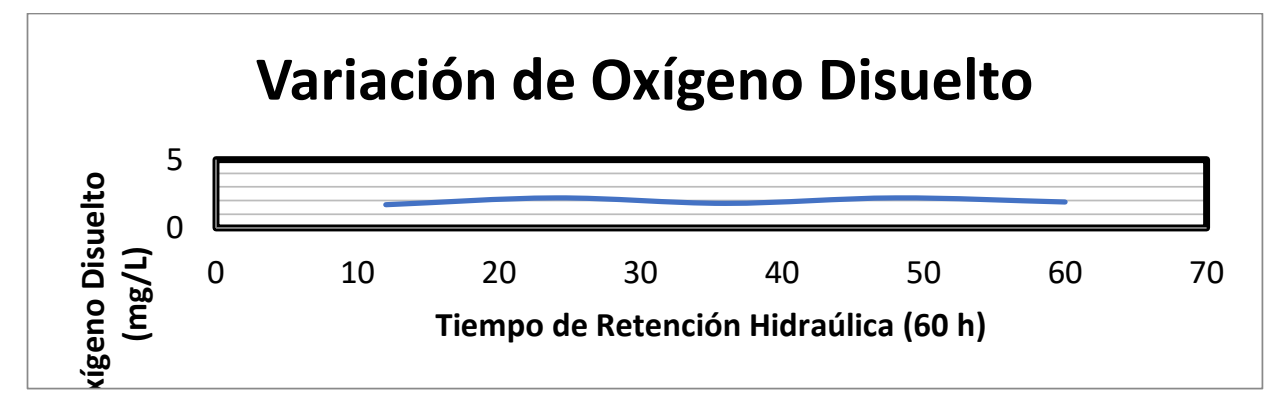

Figura 9. Variación de Oxígeno Disuelto en las 60 horas de TRH (Autores) 
Tiempo de retención hidráulica 48 horas

Tabla 15. Tiempo de Retención Hidráulica de 48 horas

\begin{tabular}{|c|c|c|c|c|}
\hline \multirow{2}{*}{$\begin{array}{c}\mathrm{N}^{\circ} \text { de } \\
\text { medicines }\end{array}$} & \multicolumn{3}{|c|}{ Parámetros } & \multirow{2}{*}{$\begin{array}{l}\text { Intervalos } \\
\text { de control }\end{array}$} \\
\hline & pH & $\begin{array}{c}\text { Temperatura } \\
{ }^{\circ} \mathrm{C} \\
\end{array}$ & OD mg/L & \\
\hline 1 & 6.20 & 21.2 & 1.9 & $\mathrm{c} / 12 \mathrm{~h}$ \\
\hline 2 & 6.45 & 20.4 & 2.2 & $\mathrm{c} / 12 \mathrm{~h}$ \\
\hline 3 & 6.83 & 20.9 & 2.1 & $\mathrm{c} / 12 \mathrm{~h}$ \\
\hline 4 & 7.20 & 20.7 & 1.9 & $\mathrm{c} / 12 \mathrm{~h}$ \\
\hline Promedios & 6.67 & 20.8 & 2025 & \\
\hline
\end{tabular}

Fuente: Grupo de Investigación.

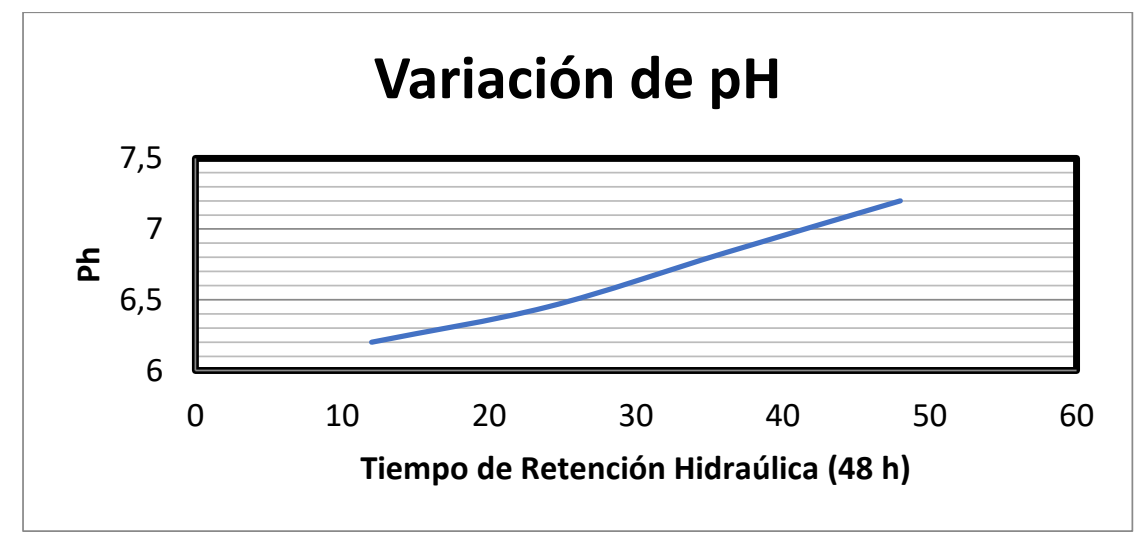

Figura10. Variación de pH en las 48 horas de TRH (Autores)

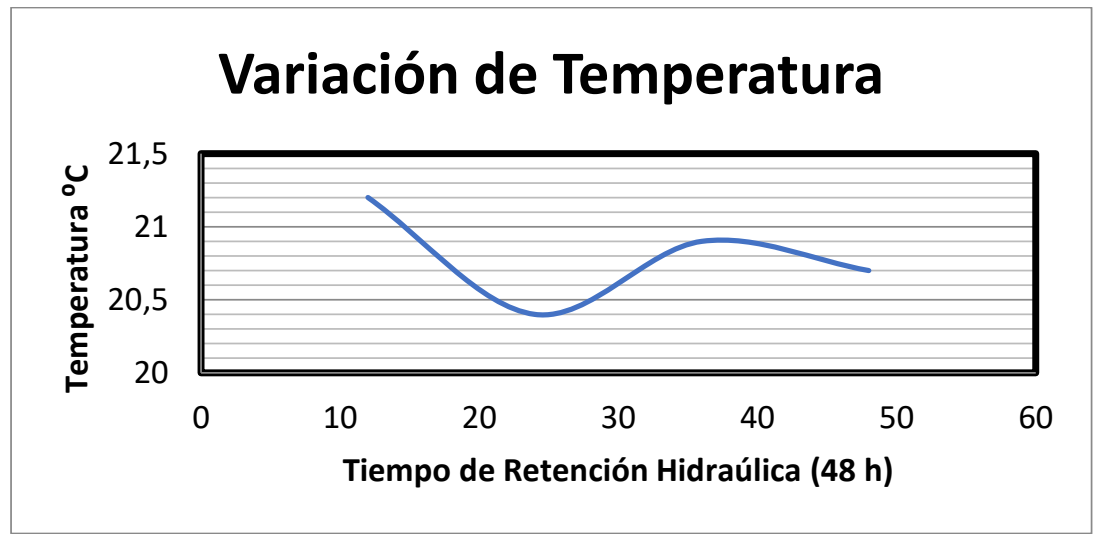

Figura11. Variación de Temperatura en las 48 horas de TRH (Autores) 


\section{Variación del Oxígeno Disuelto}

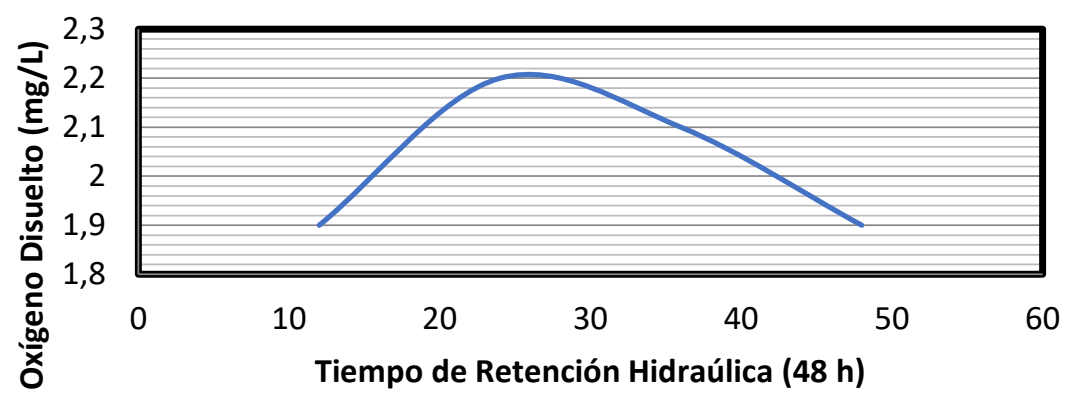

Figura12.Variación de Oxígeno Disuelto en las 48 horas de TRH (Autores)

\section{Conclusiones}

- Durante la caracterización inicial del agua residual empleada para el tratamiento se pudo determinar que ésta presenta una alta carga de materia orgánica ya que tiene valores elevados de DBO y DQO de 28300 y 32000 respectivamente.

- En base a la caracterización del agua residual se diseñó el sistema de tratamiento de agua residual por lodos activos y posteriormente se efectuó su construcción considerando el $12 \%$ de las dimensiones obtenidas en los cálculos, teniendo así una planta a escala de laboratorio con un tanque reservorio, de aireación y sedimentación de $0.5 \mathrm{~m}, 0.5 \mathrm{~m}$ y $0.75 \mathrm{~m}$ de largo, ancho y profundidad respectivamente, mientras que los tanques de almacenamiento de agua tratada y lodos residuales presentan medidas menores de $0.5 \mathrm{~m}$ de ancho, $0.5 \mathrm{~m}$ de largo y $0.5 \mathrm{~m}$ de profundidad.

- Una vez que el sistema de tratamiento fue instalado se pudo llevar a cabo el proceso de tratamiento durante un periodo de 11 días durante el cual se consideraron diferentes tiempos de retención hidráulica consiguiendo cumplir con el objetivo planteado al lograr una disminución de la carga contaminante de DBO en un $98 \%$.

- El tratamiento se logró mantener los valores de $\mathrm{pH}$, temperatura y OD adecuados para un óptimo desarrollo de los microorganismos, mediante el estricto control de los mismos.

- En asiento a la experimentación se establecieron las constantes cinéticas propias para el agua residual de la quesera "Llin Llin - Pucara" las mismas que tienen los siguientes valores: $\mathrm{K}=58.82 \mathrm{~d}-1, \mathrm{Y}=0,1215 \mathrm{mg} \mathrm{SSV} / \mathrm{mg} \mathrm{DBO}$ y $\mathrm{Kd}=2,045 \mathrm{~d}^{\cdot 1}$.

- Se determinó los valores de los coeficientes de transferencia de oxígeno (Kla) para el agua residual y potable obteniéndose los valores de $2.81 \mathrm{~h}^{-1}$ y $4.22 \mathrm{~h}^{-1}$ respectivamente.

Referencias Bibliográficas

BORJA, M. (23 de MAYO de 2011).

http://dspace.espoch.edu.ec/bitstream/123456789/1626/1/236T0043.pdf. Obtenido de http://dspace.espoch.edu.ec/bitstream/123456789/1626/1/236T0043.pdf. 
Castillo, A. M. (2014). DISEÑO E IMPLEMENTACIÓN DE UNA PTAR POR LODOS ACTIVOS A ESCALA DE LABORATORIO, FACULTAD DE CIENCIAS ESPOCH",". Riobamba.

EDDY, T CALF Y. (2012). Ingenieria sanitaria tratamiento. evacuacion. NEW YORK USA: MAC GRAW.

gobierno, e. (15 de marzo de 2014).w.w.w.epa-gov./español/. Obtenido de w.w.w.epagov./español/.

Romero, J. (2014). Tratamiento de aguas residuales. En J. Romero. Bogotá - Colombia.: , Editorial Escuela Colombiana de Ingeniería., 2014.

usal, c. (24 de noviembre de 2013). http://cidta.usal.es/curso/ETAP/modulos/libros/Caracteristicas.PDF. Obtenido de http://cidta.usal.es/curso/ETAP/modulos/libros/Caracteristicas.PDF.

Valdez, E. (2003). Ingenieria de los sistemas de tratamientos y disposiones de aguas rediduales. Mexico: Fundacion Ica.

Viracucha. (2012). Tratamiento biologico de aguas residuales generadas em un ingenio azucarero. Quito: Universidad Central del Ecuador. 


\section{PARA CITAR EL ARTÍCULO INDEXADO.}

Castillo Reinoso, A. M., Criollo Quizphi, E. C., \& Oñate Oñate, M. P. (2020). Diseño e implementación de una PTAR por lodos activos a escala de laboratorio, Facultad de Ciencias Espoch. Ciencia Digital, 4(1), 385-406. https://doi.org/10.33262/cienciadigital.v4i1.1119

\section{Ciencia \\ LDigital}

El artículo que se publica es de exclusiva responsabilidad de los autores y no necesariamente reflejan el pensamiento de la Revista Ciencia Digital.

El artículo queda en propiedad de la revista y, por tanto, su publicación parcial y/o total en otro medio tiene que ser autorizado por el director de la Revista Ciencia Digital.
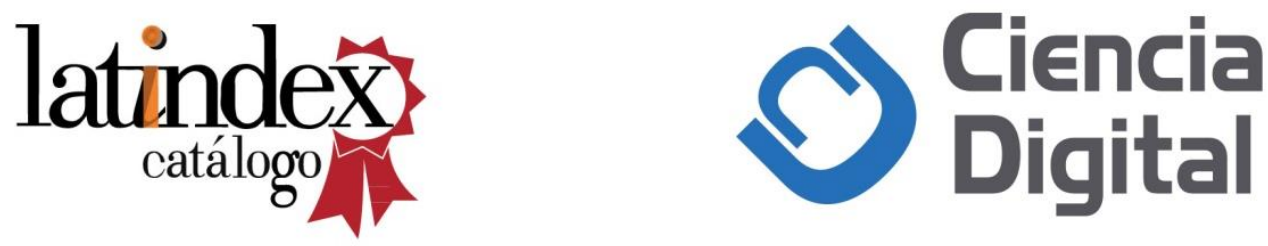